\title{
RET mutation and increased angiogenesis in medullary thyroid carcinomas
}

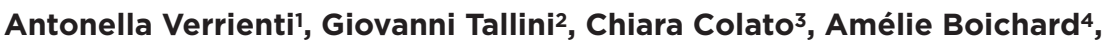 \\ Saula Checquolo5, Valeria Pecce1, Marialuisa Sponziello1, Francesca Rosignolo1, \\ Dario de Biase6, Kerry Rhoden7, Gian Piero Casadei8, Diego Russo9, Michela Visani², \\ Giorgia Acquaviva², Marco Ferdeghini ${ }^{3}$, Sebastiano Filetti ${ }^{1}$ and Cosimo Durante ${ }^{1}$
}

\author{
'Department of Internal Medicine and Medical Specialties, 'Sapienza' University of Rome, Rome, Italy \\ 2Department of Medicine (DIMES), Anatomic Pathology-Molecular Diagnostic Unit AUSL of Bologna, \\ University of Bologna School of Medicine, Bologna, Italy \\ ${ }^{3}$ Department of Diagnostics and Public Health, University and Hospital Trust of Verona, Verona, Italy \\ 4Laboratoire de Recherche Translationnelle et, Centre de Ressources Biologiques, AMMICA, \\ INSERM US23/CNRS UMS3655, Gustave Roussy, Villejuif, France \\ 5Laboratory of Molecular Pathology, Department of Medico-Surgical and Biotechnology, \\ 'Sapienza' University of Rome, Latin, Italy \\ ${ }^{6}$ Department of Pharmacology and Biotechnology (FaBiT), University of Bologna, Bologna, Italy \\ ${ }^{7}$ Department of Medicine (DIMEC), Medical Genetics Unit, University of Bologna School of Medicine, Bologna, Italy \\ ${ }^{8}$ Anatomic Pathology Unit, AUSL di Bologna-Maggiore Hospital, Bologna, Italy \\ 'Department of Health Sciences, University of Catanzaro 'Magna Graecia', Catanzaro, Italy
}

Correspondence should be addressed to A Verrienti Email

antonella.verrienti@ uniroma1.it

\begin{abstract}
Advanced medullary thyroid cancers (MTCs) are now being treated with drugs that inhibit receptor tyrosine kinases, many of which involved in angiogenesis. Response rates vary widely, and toxic effects are common, so treatment should be reserved for MTCs likely to be responsive to these drugs. RET mutations are common in MTCs, but it is unclear how they influence the microvascularization of these tumors. We examined 45 MTCs with germ-line or somatic RET mutations (RETmut group) and 34 with wildtype RET (RETwt). Taqman Low-Density Arrays were used to assess proangiogenic gene expression. Immunohistochemistry was used to assess intratumoral, peritumoral and nontumoral expression levels of VEGFR1, R2, R3, PDGFRa, PDGFB and NOTCH3. We also assessed microvessel density (MVD) and lymphatic vessel density (LVD) based on CD31-positive and podoplanin-positive vessel counts, respectively, and vascular pericyte density based on staining for a-smooth muscle actin (a-SMA), a pericyte marker. Compared with RETwt tumors, RETmut tumors exhibited upregulated expression of proangiogenic genes (mRNA and protein), especially VEGFR1, PDGFB and NOTCH3. MVDs and LVDs were similar in the two groups. However, microvessels in RETmut tumors were more likely to be a-SMA positive, indicating enhanced coverage by pericytes, which play key roles in vessel sprouting, maturation and stabilization. These data suggest that angiogenesis in RETmut MTCs may be more intense and complete than that found in RETwt tumors, a feature that might increase their susceptibility to antiangiogenic therapy. Given their increased vascular pericyte density, RETmut MTCs might also benefit from combined or preliminary treatment with PDGF inhibitors.
\end{abstract}

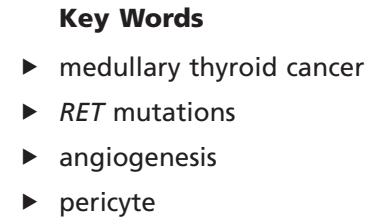

Endocrine-Related Cancer (2016) 23, 665-676 


\section{Introduction}

Medullary thyroid carcinomas (MTCs) arise from the thyroid parafollicular cells and represent $1-2 \%$ of all thyroid cancers (Wells et al. 2015). Surgical resection is the only effective treatment for these tumors (de Groot et al. 2006, Deshpande et al. 2011). Despite their relatively slow growth, MTCs are characterized by early metastatic spread, and the effective treatment of patients with advanced disease is still a major challenge (Ferreira \& Maia 2013). Indeed, mean survival for patients with MTC is lower than that associated with more common thyroid cancers, and in the presence of distant metastases, the 10-year rate drops as low as 40\% (Chau \& Haddad 2013).

Increasing knowledge of the distinct signaling pathways and genetic abnormalities involved in the pathogenesis of cancer has allowed the development of targeted molecular therapies (Ferreira \& Maia 2013). Most MTCs are sporadic cancers, but a hereditary pattern is discernible in $25-30 \%$ of cases (Pusztaszeri et al. 2014). Ninety-nine percent of these familial cases are caused by germ-line mutations in the receptor tyrosine kinase (RTK) gene RET. RET mutations are less common in sporadic MTCs, roughly half of which harbor somatic alterations of this gene (Wu et al. 2011). In the last few years, somatic mutations in the $R A S$ oncogenes have also been identified in sporadic MTCs, with reported prevalence rates ranging from $11 \%$ (Ciampi et al. 2013) to $44 \%$ (Boichard et al. 2015). Nevertheless, in a small proportion of sporadic MTCs, no genetic alterations are detected.

Small-molecule inhibitors of receptor tyrosine kinases (TKIs) are one of the most promising approaches for treating MTCs. Indeed, encouraging results have emerged from clinical trials in which these drugs were tested in MTC (Ferreira \& Maia 2013). The TKIs most widely investigated in MTC patients are drugs that target multiple RTKs, including those involved in angiogenesis like VEGFRs and PDGFRs. The efficacy of these agents varies widely, with partial responses reported in $0-40 \%$ of MTC patients and disease stabilization in 27-55\% (Ferreira \& Maia 2013). Unfortunately, TKIs also interact with receptors that mediate physiological functions and are thus associated with a number of highly toxic side effects. Careful advance selection of the patients likely to be responsive to these drugs is, therefore, essential. In-depth analysis of the molecular alterations present in the tumor is fundamental for selecting the most effective drug treatment. For example, MTC response rates to vandetanib, one of the most promising TKIs, are higher in RET M918T mutation-positive patients than in those without this mutation, and patients with RET-activating mutations at codon 804 (V804L, V804M) are resistant to vandetanib and some other TKIs as well (Ferreira \& Maia 2013).

Like most endocrine tumors, MTC are richly vascularized, and impairment of tumor vascularization may be at least one of the mechanisms underlying the TKIs' therapeutic effects in these tumors. However, it is not clear whether specific molecular alterations in MTCs influence the features of angiogenesis and tumor microvascularization in MTC and, if so, what impact they have on the response to TKI therapy. To address these questions, we conducted an analysis of the genetic alterations and features of tumor microvascularity in a large series of MTCs.

\section{Methods}

\section{Patients, tissues and cell lines}

This study was approved by the research ethics committees of all participating centers. Written informed consent was obtained from each enrolled tissue donor. Unless otherwise specified, commercial products were used in accordance with the manufacturer's instructions.

A total of 79 MTCs were examined. The 53 tumors for which fresh frozen tissue samples were available were used for gene expression analyses. These fresh frozen tissue samples were also subjected to mutation analysis, along with formalin-fixed paraffin-embedded (FFPE) samples from 26 other tumors (total: 79). Immunohistochemical analyses were performed on all FFPE samples (representing a total of 35 MTCs) and on a tissue microarray containing samples of 30 other tumors. The array was constructed according to standard procedures and included three separate cores (diameter: $0.6 \mathrm{~mm}$ ) from each MTC. Histology sections from all tumor specimens (snapfrozen and FFPE) were reviewed by two pathologists with experience in thyroid disease (G T and G P C), who confirmed the diagnosis of MTC and the adequacy of the neoplastic cell population in each sample analyzed.

TT cells were purchased from the American Type Culture Collection (Manassas, VA, USA). They are derived from a primary MTC and harbor a cysteine 634 to tryptophan (C634W) mutation in RET exon 11. TT cells were grown in Ham's F12 Medium with 10\% fetal bovine serum (FBS) (Gibco). The MZ-CRC1 cell line (kindly provided by Dr M Santoro) harbors a methionine 918 to tryptophan (M918T) mutation in RET exon 16. These cells were grown in DMEM with 10\% FBS. The media used

Published by Bioscientifica Ltd 
for both cultures were supplemented with $2 \mathrm{mmol} \mathrm{L}^{-1}$ glutamine and $100 \mathrm{U} / \mathrm{mL}$ penicillin-streptomycin (Gibco).

\section{Sanger sequencing}

FFPE or fresh frozen tumor tissues from each MTC were subjected to Sanger sequencing to identify mutations involving the RET (exons 10, 11, 13, 14, 15, and 16), $H$-RAS, K-RAS and N-RAS genes (exons 2, 3 and 4). DNA was extracted using the NucleoSpin Tissue Kit (MachereyNagel GmbH \& Co. KG, Düren, Germany).

Primers and PCR conditions used for these analyses have been described elsewhere (Castellone et al. 2010, Puppin et al. 2014). Amplicons were purified with the NucleoSpin Gel and PCR Clean-up Kit (Macherey-Nagel GmbH \& Co. KG Düren, Germany) and sequenced with the BigDye Terminator Cycle Sequencing Kit, version 3.1 (Thermo Fisher Scientific). Sequencing reactions were purified using the NucleoSEQ Kit (Macherey-Nagel) and loaded onto an ABI 3130xl Genetic Analyzer (Thermo Fisher Scientific). Data were analyzed with Sequencing Analysis software, version 5.3 (Thermo Fisher Scientific). Each mutation was confirmed twice, starting from the PCR step. Somatic and germ-line RET mutations were distinguished on the basis of DNA sequencing data of nontumor thyroid tissue samples from the same patient.

\section{Angiogenic gene expression in fresh frozen tumor tissues}

Total RNA was extracted from fresh frozen tumor tissues and reverse-transcribed, as described previously (Puppin et al. 2014, Sponziello et al. 2014). Angiogenic gene transcript levels were measured with real-time PCR performed with custom-designed microfluidic cards (Taqman Low-Density Arrays [TLDAs], Thermo Fisher Scientific), each configured with the specific Taqman Gene Expression Assay (Thermo Fisher Scientific) for the main components of the Notch pathway (receptors: NOTCH1 , 2, 3, 4; ligands: DLL1, DLL3, DLL4, JAG1, JAG2; regulators: the gamma-secretases PSEN1 and 2, DTX1, MAML1 and RBPJ; effectors: HES1, HEY1-2-L; the repressor $N U M B$ ); the vascular endothelial growth factor (VEGF) pathway (ligands: VEGFA and VEGFC; receptors VEGFR1, 2 and 3 and NRP1); and the platelet-derived growth factor (PDGF) pathway (ligands: PDGFA, PDFGB; receptors: PDGFR a and PDGFRb); as well as for the following genes: angiopoietin-1 and -2, MMPS 1-2-3-9, and TIMPS 1-2-3. Three housekeeping genes (GAPDH, GUSB and B2M) were included in each run for data normalization purposes.
The reactions were set up as described previously (Sponziello et al. 2014), and the amplifications were performed using a 7900HT Fast Real-Time PCR System (Thermo Fisher Scientific). Ct values were calculated with SDS 2.3 software (Thermo Fisher Scientific), and the detection threshold was set at 33. Data were analyzed with RQ Manager 1.2 software (Thermo Fisher Scientific). GAPDH was selected as the endogenous control gene because of its stability in the biological replicates. Results were determined by the $2^{-\Delta \Delta C t}$ method (Puppin et al. 2014) and expressed as relative expression normalized to a calibrator sample.

\section{Immunohistochemical analysis of angiogenic marker expression and microvessel and lymphatic vessel densities}

Immunohistochemistry was used to obtain proteinlevel verification of the results of the angiogenic gene expression analyses described above. These studies were performed according to standard procedures (Tallini et al. 1998) using a BenchMark XT automated platform (Ventana Medical Systems, Tucson, AZ, USA) and biotinfree detection procedures. Angiogenic protein expression was analyzed in 30 FFPE tumor sections and in tissue microarray sections representing 35 MTCs. In each section, we scored the intensity of immunoreactivity in individual cells (weak $=1$, moderate $=2$, strong $=3$ ) and the percentage of immunoreactive cells $(<5 \%$ positive cells $=0,5-24 \%$ positive cells $=1,25-75 \%$ positive cells $=2$, $>75 \%$ positive cells $=3$ ). The product of these two scores provided a semiquantitative index of expression (range: $0-9$ ). For each analyzed protein, labeled cells were counted in three areas: tumor tissue (zone T), peritumoral tissue (i.e. $<2 \mathrm{~mm}$ from the tumor) (zone $\mathrm{T} / \mathrm{N}$ ) and nontumoral tissue ( $>2 \mathrm{~mm}$ from the tumor) (zone $\mathrm{N}$ ).

Microvessel and lymphatic vessel densities (MVD and LVD, respectively) were evaluated in FFPE sections using the Weidner protocol with minor modifications, as reported previously (Durante \& Filetti 2011). For assessment of MVD, vascular endothelial cells were identified with anti-CD31 antibodies; for LVD studies, lymphatic endothelial cells were identified with antibodies against podoplanin. FFPE sections were also stained with antibodies against $\alpha$ smooth muscle actin (a-SMA), a phenotypic marker used to identify pericytes. CD31-, podoplanin- and a-SMA-positive microvessels were counted in zones $\mathrm{T}, \mathrm{T} / \mathrm{N}$ and $\mathrm{N}$.

Antibodies Mouse monoclonal antibody against VEGFR1 (dilution 1:500), rabbit polyclonal antibody

Published by Bioscientifica Ltd. 
against PDGFB (1:100) and mouse monoclonal antibody against NOTCH3 (1:100) were obtained from Abcam. Rabbit monoclonal antibody against VEGFR2 (1:500) and rabbit monoclonal antibody against PDGFRa (1:500) were from Cell Signaling, and mouse monoclonal antibody against VEGFR3 (KLT9 clone) (1:50) was purchased from Novocastra-Leica Biosystems (Newcastle, UK). Antibodies against CD31 (JC70 clone), podoplanin (D2-40 clone) and a-SMA (1A4 clone) were purchased from Ventana Medical Systems, Tucson, AZ, USA).

\section{Short interfering RNA (siRNA) silencing of RET in MTC cell lines}

TT and MZ-CRC1 cells were plated at 40\% confluence in $35 \mathrm{~mm}$ dishes containing Ham's F12 Medium (TT cells) or DMEM (MZ-CRC1 cells) supplemented with $4 \%$ FBS. Twenty-four hours later, cells were transfected with $100 \mathrm{nmol} \mathrm{L}^{-1}$ duplex siRNAs (ON-target plus SMARTpool) against human RET (siRET; \# L-003170-00) (Dharmacon, Lafayette, CO, USA) using Dharmafect reagent. Control cells (CTL) were transfected with a nontargeting pool of siRNA (\# D-001206-13-05). Twenty-four and forty-eight hours after transfection, cells were harvested for analysis of angiogenic gene expression, as described below.

Angiogenic gene expression analysis RNA for these assays was isolated with the RNeasy kit (Qiagen). The RNA concentration was measured with a NanoDrop Spectrophotometer (Thermo Fisher Scientific). RNA was retrotranscribed using the High Capacity cDNA Reverse Transcription kit (Thermo Fisher Scientific). Complementary DNA was analyzed with Taqman Gene Expression Assays-on-Demand (Thermo Fisher Scientific) to quantify the expression of VEGFA, VEGFR2, PDGFA, PDGFB, PDGFRa, PDGFRb, NOTCH1, NOTCH3, JAG1, JAG2, DLL4, HES1, HES5, HEY1, HEY2 and HEYL genes. The GADPH gene was used as an endogenous reference. Results (determined with the $2^{-\Delta \Delta \mathrm{Ct}}$ method) were normalized to corresponding calibrator samples and expressed as mean \pm s.D. of three replicates.

Western blotting analysis and Human PhosphoRTK array Total protein was extracted from TT and MZ-CRC1 cells using a buffer consisting of $150 \mathrm{mmol} \mathrm{L}^{-1}$ Tris- $\mathrm{HCl} \mathrm{pH} 7.4,50 \mathrm{mmolL}^{-1} \mathrm{NaCl}$, Triton 1\%, EDTA $20 \mathrm{mmolL}^{-1}$, glycerol 5\%, protease inhibitor cocktail (PIERCE, Thermo Fisher Scientific), $2 \mathrm{mmolL}^{-1}$ PMSF and $2 \mu \mathrm{g} / \mathrm{mL}$ leupeptin. RET protein was detected with monoclonal anti-RET antibody (Cell Signaling \#3220) produced in rabbits (dilution 1:1000). Phosphorylated and total ERK protein were detected with monoclonal anti-phospho-ERK1/2 (Thr202/Tyr204) antibody (Cell Signaling \#4370) and with monoclonal anti-ERK1/2 antibody (Cell Signaling \#4695), both produced in rabbits (dilution 1:2000 and 1:1000).

Phosphorylated and total AKT proteins were detected with anti-phospho-AKT (Ser473) antibody (Cell Signaling \#4058) and with anti-AKT antibody (Cell Signaling \#9272), both produced in rabbits (dilution 1:1000). JAGGED1 protein was detected with anti-JAGGED1 antibody (Santa Cruz sc-8303) produced in rabbits (dilution 1:1000). DLL4 protein was detected with polyclonal anti-DLL4 antibody (Abcam ab176876) produced in rabbits (dilution 1:1000). Tubulin was detected with monoclonal antitubulin antibody (Santa Cruz sc-5286) produced in mice (dilution 1:5000).

The protein lysates were also hybridized on Human Phospho-RTK array membranes (R\&D Systems) to assess the phosphorylation levels of RET, VEGFRs and PDGFRs. The average signal (pixel density) of the pair of duplicate spots of each RTK was normalized to the negative control spots used as background value. Phosphorylated RTKs were detected using chemiluminescence with Clarity Western ECL substrate (BIO-RAD) and a charge-coupled device camera (Chemidoc, Bio-Rad). Data were analyzed with Image Lab software (Bio-Rad).

\section{Statistical analysis}

All statistical analyses were carried out with GraphPad Prism software, version 5.0 (GraphPad Software). TLDA results are expressed as mean \pm s.D. The Mann-Whitney test was used to evaluate intergroup differences. The results of real-time PCR analysis of TT and MZ-CRC1 cell lines were expressed as group mean \pm s.D., and intergroup differences were analyzed with the $t$-test. Phospho-RTK array data were expressed as mean pixel density \pm s.D., and differences were assessed with a t-test. IHC scores were analyzed as follows: intergroup differences in CD31, podoplanin and a-SMA expression were analyzed with one-way RM ANOVA and two-way RM ANOVA with the Bonferroni multiple comparison post-test. Intergroup differences in the expression levels of all other proteins were assessed with the Friedman test or the Kruskal-Wallis test, each used with Dunn's multiple comparisons test. $P$ values $<0.05$ were considered statistically significant.

Published by Bioscientifica Ltd 


\section{Results}

\section{RET and RAS mutational profiles of the 79 MTCs}

The RET/RAS mutational profiles of the 79 MTCs are summarized in Supplementary Table 1, see section on supplementary data given at the end of this article. On the basis of these findings, 45 MTCs were classified as RET-mutant (RETmut), 23 as RASmut tumors and the remaining 11 were characterized by wild-type (wt) forms of both genes (RETwt/RASwt tumors).

Table 1 Expression levels of genes involved in angiogenesis and $R E T$ status.

\begin{tabular}{|c|c|c|c|}
\hline & $\begin{array}{c}\text { RETwta } \\
n=20\end{array}$ & $\begin{array}{c}\text { RETmutb } \\
n=33\end{array}$ & $P$ value \\
\hline VEGFA & $1 \pm 1.22$ & $1.37 \pm 0.67$ & $<0.01$ \\
\hline VEGFC & $1 \pm 1.03$ & $1.40 \pm 1.30$ & NS \\
\hline VEGFR1 & $1 \pm 0.82$ & $1.88 \pm 1.34$ & $<0.01$ \\
\hline VEGFR2 & $1 \pm 0.74$ & $2.21 \pm 1.33$ & $<0.001$ \\
\hline VEGFR3 & $1 \pm 0.96$ & $1.64 \pm 1.00$ & $<0.05$ \\
\hline$N R P 1$ & $1 \pm 0.55$ & $1.50 \pm 0.89$ & $<0.05$ \\
\hline PDGFA & $1 \pm 0.59$ & $1.80 \pm 1.13$ & $<0.01$ \\
\hline$P D G F B$ & $1 \pm 0.55$ & $1.82 \pm 1.27$ & $<0.01$ \\
\hline PDGFRa & $1 \pm 1.20$ & $5.55 \pm 5.73$ & $<0.001$ \\
\hline PDGFRb & $1 \pm 0.70$ & $2.51 \pm 1.62$ & $<0.001$ \\
\hline NOTCH1 & $1 \pm 0.78$ & $1.98 \pm 1.54$ & $<0.01$ \\
\hline NOTCH2 & $1 \pm 1.03$ & $1.88 \pm 1.37$ & $<0.01$ \\
\hline NOTCH3 & $1 \pm 0.80$ & $2.27 \pm 1.57$ & $<0.01$ \\
\hline NOTCH4 & $1 \pm 0.83$ & $2.15 \pm 1.50$ & $<0.01$ \\
\hline$D L L 1$ & $1 \pm 0.77$ & $1.01 \pm 0.50$ & NS \\
\hline DLL3 & $1 \pm 1.60$ & $1.20 \pm 1.38$ & NS \\
\hline$D L L 4$ & $1 \pm 0.52$ & $1.61 \pm 1.41$ & $<0.05$ \\
\hline JAG1 & $1 \pm 1.07$ & $1.41 \pm 0.74$ & $<0.01$ \\
\hline JAG2 & $1 \pm 0.66$ & $1.79 \pm 1.31$ & $<0.01$ \\
\hline PSEN1 & $1 \pm 0.39$ & $1.24 \pm 0.43$ & NS \\
\hline PSEN2 & $1 \pm 0.43$ & $1.40 \pm 0.58$ & $<0.05$ \\
\hline$D T X 1$ & $1 \pm 1.31$ & $1.44 \pm 2.35$ & NS \\
\hline$R B P J$ & $1 \pm 0.77$ & $1.27 \pm 0.59$ & $<0.05$ \\
\hline HES1 & $1 \pm 0.57$ & $1.51 \pm 1.15$ & NS \\
\hline HEY1 & $1 \pm 0.47$ & $2.01 \pm 1.43$ & $<0.01$ \\
\hline HEY2 & $1 \pm 0.53$ & $2.05 \pm 1.47$ & $<0.01$ \\
\hline$H E Y L$ & $1 \pm 0.70$ & $2.53 \pm 1.81$ & $<0.01$ \\
\hline MAML1 & $1 \pm 0.43$ & $1.68 \pm 0.84$ & $<0.01$ \\
\hline NUMB & $1 \pm 0.41$ & $1.27 \pm 0.44$ & NS \\
\hline ANGPT1 & $1 \pm 0.87$ & $1.60 \pm 1.42$ & NS \\
\hline ANGPT2 & $1 \pm 1.07$ & $2.06 \pm 1.77$ & $<0.01$ \\
\hline MMP2 & $1 \pm 1.19$ & $2.06 \pm 1.91$ & $<0.01$ \\
\hline MMPg & $1 \pm 3.43$ & $0.73 \pm 2.75$ & NS \\
\hline TIMP1 & $1 \pm 0.60$ & $1.18 \pm 0.42$ & NS \\
\hline TIMP2 & $1 \pm 1.40$ & $0.71 \pm 0.37$ & NS \\
\hline TIMP3 & $1 \pm 1.27$ & $1.28 \pm 1.27$ & NS \\
\hline
\end{tabular}

alncludes all MTCs with wt RET (regardless of RAS status); blncludes all MTCs with RET mutations (regardless of RAS status); cMann-Whitney. Results are calculated with $2-\Delta \Delta C t$ method and expressed as mean \pm s.D. normalized to RETwt group, used as calibrator sample.

\section{Expression of angiogenesis-related genes in MTCs}

The expression of 36 angiogenesis-related genes was analyzed in fresh frozen tissue samples of 53 MTCs. As shown in Table 1 , comparison of RETmut $(n=33)$ and RETwt $(n=20)$ tumors revealed significant upregulation in the former group of almost all the genes considered. Next, we compared expression levels for the same 36 genes in the RETmut and RASmut subgroups, using the RETwt/RASwt subgroup as a calibrator (Supplementary Table 2). This analysis revealed that the diffuse angiogenesis-related gene upregulation observed in the RETmut tumors (Table 1) was largely due to the differences in gene expression between the RETmut and RASmut subgroups (Supplementary Table 2).

\section{RET induction of angiogenic factors}

To confirm the link between RET oncogene activation and the overexpression of important factors involved in angiogenesis, we transfected two stable human MTC cell lines with RET antisense RNA (siRET). The strongest effect of siRET on TT and MZ-CRC1 cells was observed $48 \mathrm{~h}$ after treatment, when there were significant reductions in both RET mRNA and protein levels (Supplementary Fig. 1A, B, C, and D). The efficacy of RET knockdown at $48 \mathrm{~h}$ was further confirmed by a decrease in RET phosphorylation (Supplementary Fig. 1E and G) with consequent reduction in phosphorylated ERK and AKT (pERK and pAKT, respectively) (Supplementary Fig. $1 \mathrm{~F}$ and $\mathrm{H}$ ). Subsequent analyses were, therefore, performed on cells harvested at this post-transfection time point.

Our next goal was to see how RET knockdown affected the expression of genes playing key roles in the VEGF, PDGF and Notch pathways, all of which were significantly dysregulated in the RETmut MTCs we analyzed (Table 1). As shown in Fig. $1 \mathrm{~A}$ and $\mathrm{B}$, transcription of VEGFA, VEGFR2 and $P D G F R b$ was significantly downregulated in both cell lines $48 \mathrm{~h}$ post-transfection. TT cells also displayed significant reductions in PDGFRa and PDGFB expression. Receptor phosphorylation was analyzed to assess the activation state of VEGF and PDGF pathways. Levels of pVEGFR2, pVEGFR3 and pPDGFRb were reduced in both cell lines (Fig. 1C and D). As shown in Fig. 1E and F, analysis of genes encoding Notch pathway components revealed no significant change after RET knockdown in the expression of NOTCH effector genes (except for HES5 in MZ-CRC1 cell line). To explore the possibility that RET

Published by Bioscientifica Ltd 

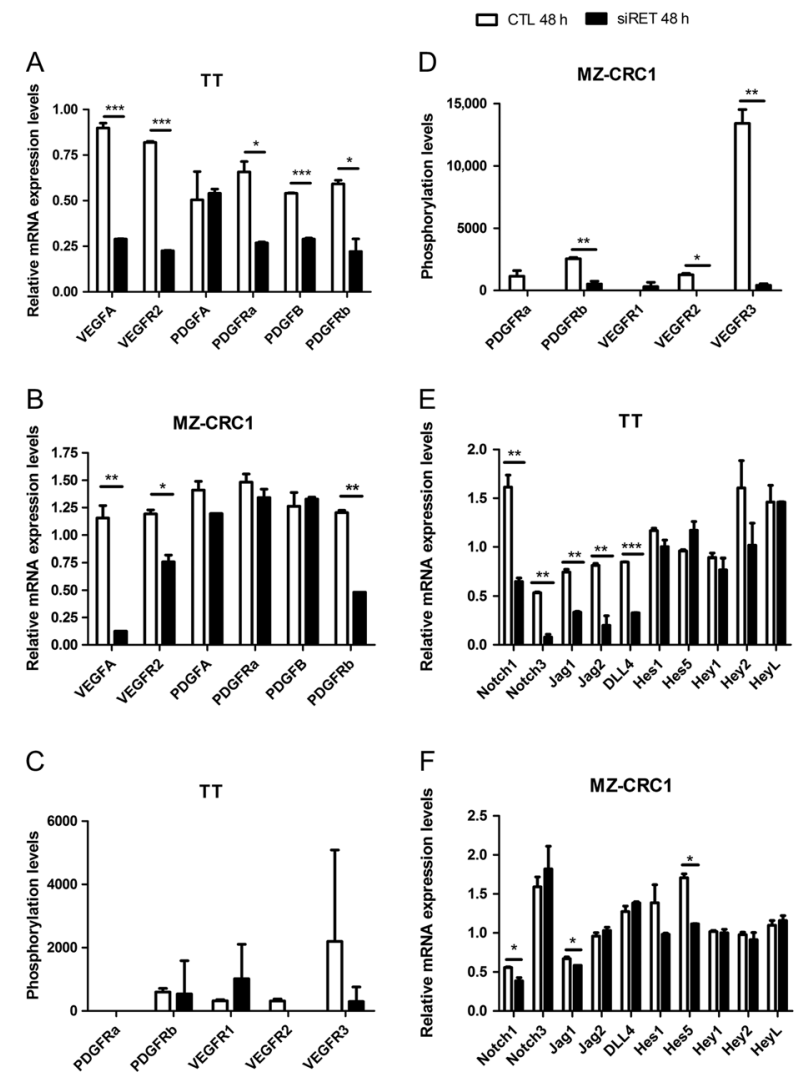

activation could contribute to the activation of Notch signaling in other cells of the tumor (e.g. endothelial cells in the tumor microenvironment), we analyzed the expression of DLL4 and JAG1, two of the NOTCH ligands that appeared to be significantly dysregulated in the RETmut MTC (Table 1). Notably, JAG1 protein levels decreased appreciably after RET silencing in the MZ-CRC1 cells, and DLL4 expression diminished in both cell lines (Fig. 1G).
G

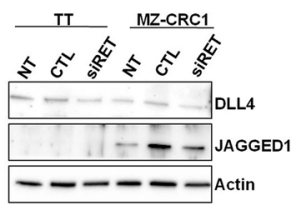

\begin{abstract}
Figure 1
Gene and protein expression analysis in MTC cell lines after RET knockdown. TT and MZ-CRC1 cells were transfected with siRET or with a nontargeting pool of siRNAs (CTL) and analyzed $48 \mathrm{~h}$ later. Untreated cells (NT) were used as calibrators (data not shown). Panels A and B: mRNA levels (in TT and MZ-CRC1 cells, respectively) for genes encoding VEGF and PDGF pathway components. Panels C and D: VEGFR and PDGFR phosphorylation levels determined with the Human Phospho-RTK array. Data are expressed as average signal (pixel density) of the pair duplicate spots, normalized to negative control spots used as background value. Panels $\mathrm{E}$ and F: mRNA levels (in TT and MZ-CRC1 cells, respectively) for genes encoding Notch pathway components. Panel G: Protein expression levels in both cell lines of Notch ligands DLL4 and JAG1 before and after RET knockdown. Data were analyzed with unpaired $t$ test $\left({ }^{*} P<0.05\right.$, $* * P<0.01, * * * P<0.001)$.
\end{abstract}

IHC analysis of proangiogenic protein expression and of microvessel, lymphatic vessel and vascular pericyte density in MTC

To verify the results of the angiogenic gene expression analysis shown in Table 1, we assessed the protein-level expression of six of the genes that were upregulated in RETmut MTCs. Given the limited availability of tumor specimens, we restricted this analysis to the proteins most likely to provide a comprehensive picture of in vivo
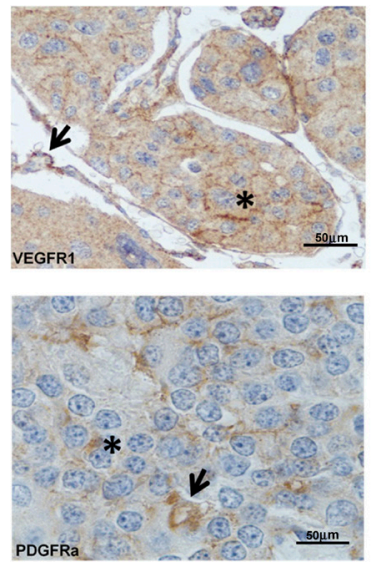
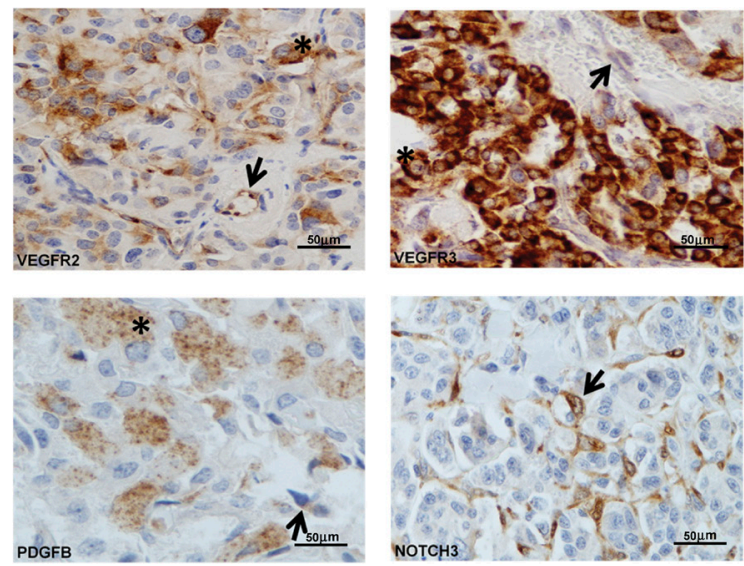

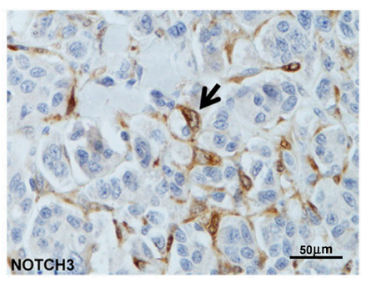

Figure 2

Representative results of immunohistochemical staining of six proangiogenic proteins in MTCs showing the localization of each protein in tumor cells (*) and/or in endothelial cells (arrows). Magnification: 400x. A full colour version of this figure is available at http://dx.doi.org/10.1530/ ERC-16-0132. 
angiogenesis: VEGFR-1, $-2,-3$ and PDGFRa, the main receptors triggering angiogenic signaling, and PDGFB and NOTCH3, which are involved in pericyte recruitment, the final step of angiogenesis. IHC analysis of 63 FFPE tumor samples (43 RETmut, 15 RASmut, 5 RETwt/RASwt) revealed no genotype-related differences in the cellular (tumor vs endothelial cell) or subcellular localization of any of the proangiogenic proteins analyzed. Three of the receptors (VEGFR1, VEGFR3 and PDGFRa) and PDGFB (the only ligand considered) were more strongly expressed in tumor cells. VEGFR2 was expressed predominantly in endothelial cells, and NOTCH3 was expressed exclusively by these cells. VEGF receptors 1,2 and 3 ,
PDGFRa and NOTCH3 were found in the cytoplasm and plasma membrane. The expression of PDGFB ligand was predominantly cytoplasmic. A representative IHC image is shown in Fig. 2.

When all the samples were analyzed (independently from their mutational status), all angiogenic proteins displayed significantly higher mean expression scores in zone $\mathrm{T}$ (tumor tissue) than in non-neoplastic tissue (zone N) (Fig. 3). For VEGFR1, VEGFR2, PDGFB and NOTCH3 (but not VEGFR3 or PDGFRa), the mean scores for zone $\mathrm{T} / \mathrm{N}$ were also significantly higher than those of zone $\mathrm{N}$. The analyses were then repeated for the RETmut vs RETwt subgroups (Fig. 4). T-zone scores were all appreciably

$\circ \mathrm{T} \quad \mathrm{T} / \mathrm{N} \diamond \mathrm{N}$

A

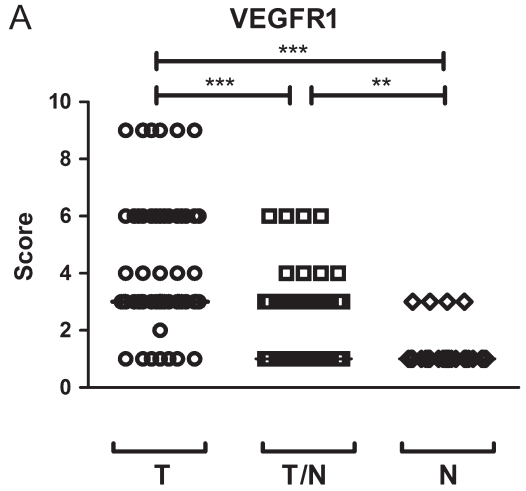

B

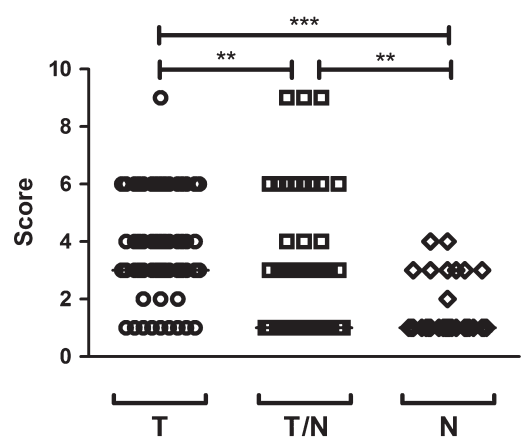

C

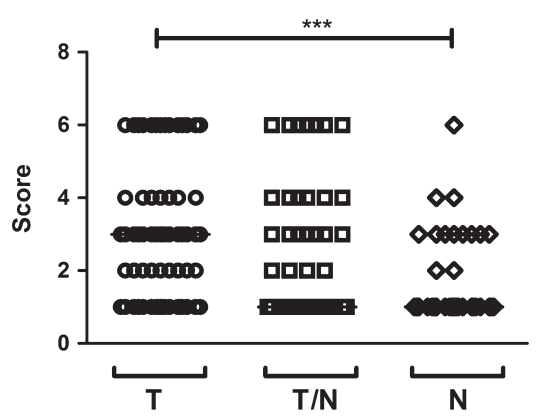

$\mathrm{D}$

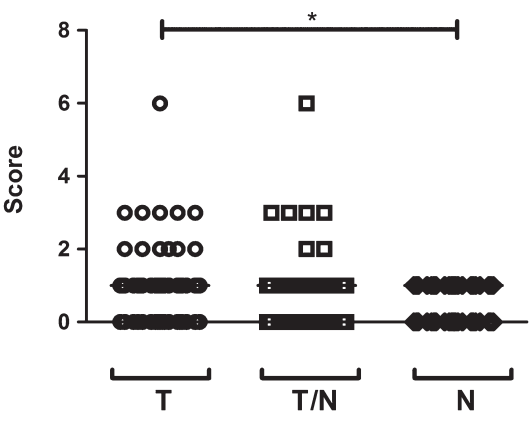

$E$

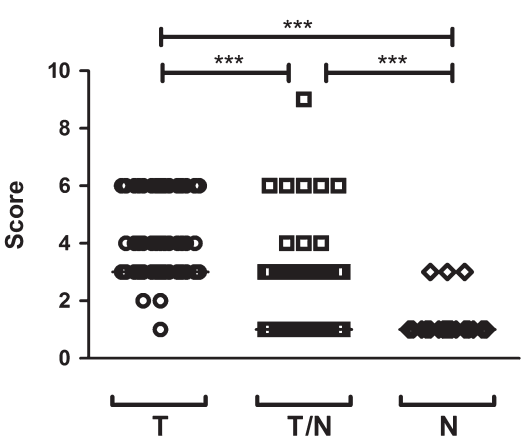

$\mathrm{F}$

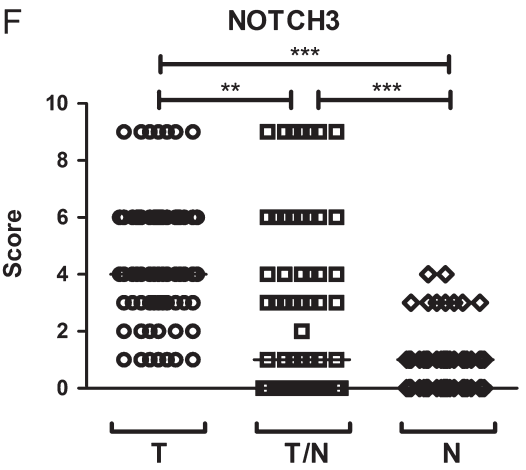

Figure 3

Immunohistochemical scores of proangiogenic protein expression (A, VEGFR1; B, VEGFR2; C, VEGFR3; D, PDGFRa; E, PDGFB; F, NOTCH3) in all 63 MTC samples by zone: T, tumor; T/N peritumoral non-neoplastic tissues (vessels located within $2 \mathrm{~mm}$ of the tumor front are considered peritumoral); $\mathrm{N}$, non-neoplastic tissues at a distance $(>2 \mathrm{~mm})$ from the tumor. Friedman test with Dunn's multiple comparison test. ${ }^{*} P<0.05, * * P<0.01, * * * P<0.001$ 
- RETwt a RETmut

A

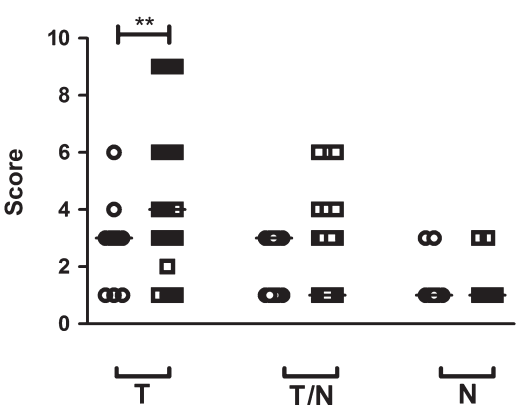

B

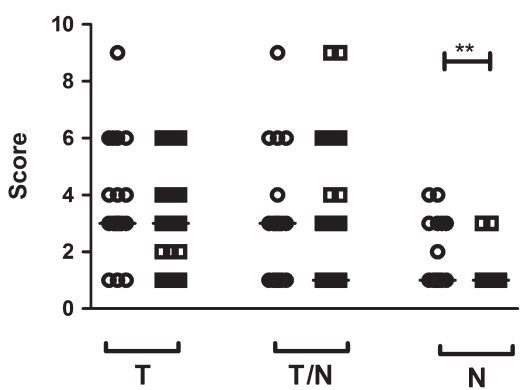

C

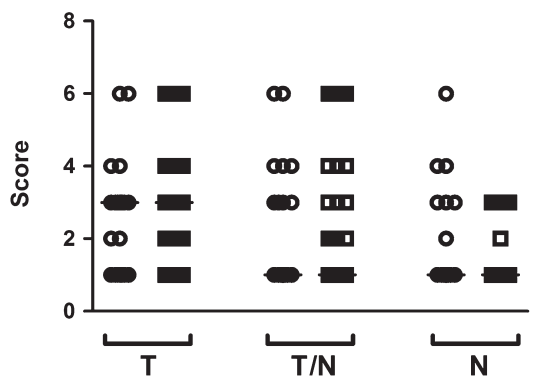

D

PDGFRa

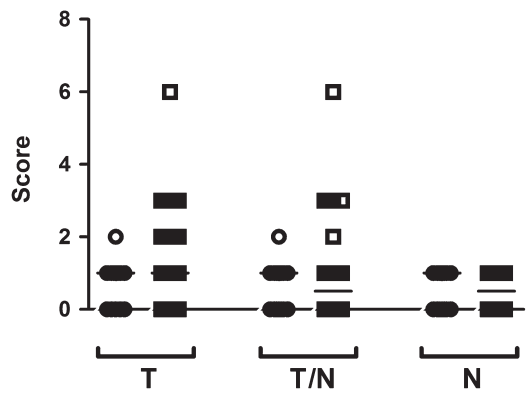

E

PDGFB

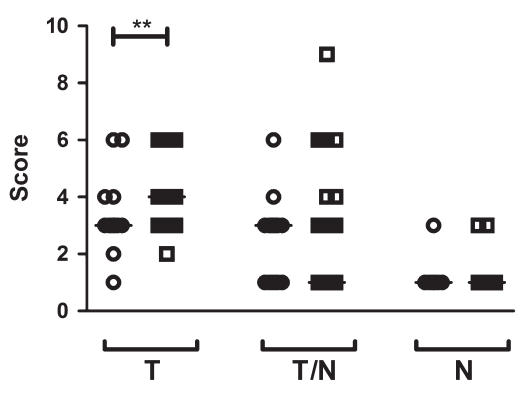

$\mathrm{F}$

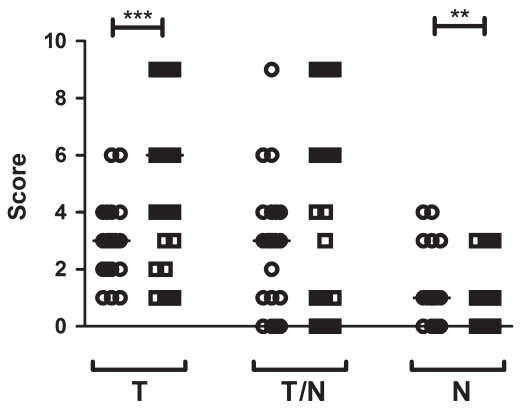

Figure 4

IHC analysis of proangiogenic protein expression in zones T, T/N and N of RETmut vs RETwt MTCs. Kruskal-Wallis test with Dunn's test for multiple comparisons: $* * P<0.01,{ }^{* * * P}<0.001$.

higher in sections from RETmut tumors, but the difference was significant only for VEGFR1, PDGFB and NOTCH3. (As shown in Supplementary Fig. 2, these findings were mainly due to differences between RETmut and RASmut tumors and, in the case of NOTCH3, also between RETmut and RETwt/RASwt tumors.) $\mathrm{T} / \mathrm{N}$ scores were similar in the three groups of tumors (Fig. 4 and Supplementary Fig. 2).

Microvessel density (CD31 staining), lymphatic vascular density (podoplanin staining) and vascular pericyte density (a-SMA staining) were assessed in 28 MTCs (4 RETwt/RASwt, 10 RASmut and 14 RETmut with somatic mutations). MTCs were highly vascular. CD31positive endothelial cells were more numerous inside the tumor; their density decreased at the interface between the tumor and the non-neoplastic peritumoral tissue $(\mathrm{T} / \mathrm{N})$
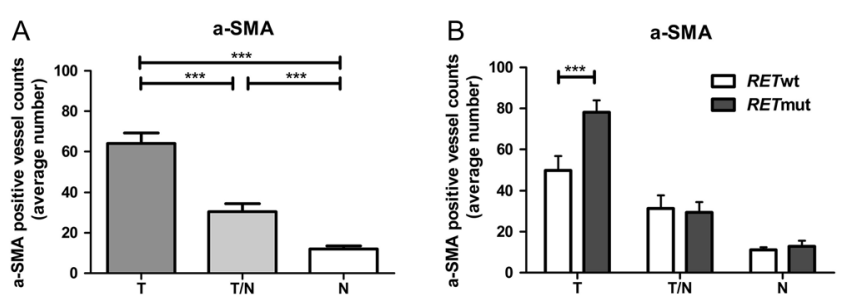

Figure 5

Location of a-SMA-positive vessels in all MTCs (A) and in tumor subgroups defined by RET status (B). T, tumor; T/N peritumoral non-neoplastic tissues (vessels located within $2 \mathrm{~mm}$ of the tumor front are considered peritumoral); $\mathrm{N}$, non-neoplastic tissues at a distance $(>2 \mathrm{~mm})$ from the tumor. Bars indicate mean numbers of a-SMA positive vessels; whiskers indicate standard error. $* * *<0.001$. Differences were assessed with one-way RM ANOVA (panel A) or two-way RM ANOVA with Bonferroni's post-test (panel B).

Published by Bioscientifica Ltd 

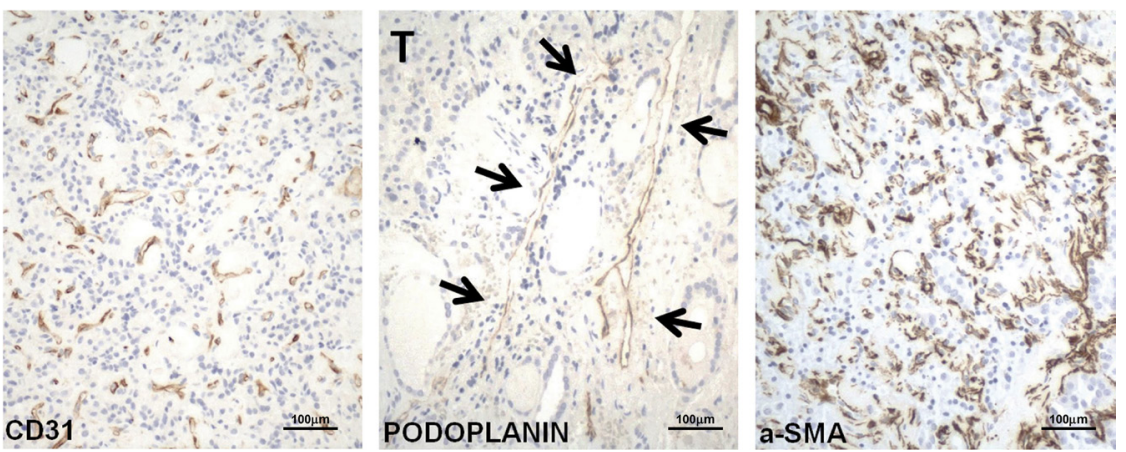

Figure 6

Immunohistochemical analysis of vascular density in an RET-mutated MTC. CD31-positive vessels were counted to estimate microvascular density, podoplanin-positive vessels were counted to estimate lymphatic vascular density and $\alpha$ smooth muscle actin (a-SMA)-positive vessels were counted to assess vascular pericyte density. In the figure immunostained with the anti-podoplanin antibody, arrows indicate the interface between the tumor $(T)$ and the surrounding non-neoplastic peritumoral tissue $(\mathrm{N})$; the figures immunostained with CD31 and a-SMA antibodies show only intratumoral vessels. Magnification: $200 x$. A full colour version of this figure is available at http://dx.doi.org/10.1530/ERC-16-0132.
(Supplementary Fig. 3A). Podoplanin-positive lymphatic endothelial cells were virtually absent within the tumor but very abundant in zone T/N (Supplementary Fig. 3B). RETmut and RETwt tumors were not significantly different in terms of intratumoral CD31-positive vessel counts or peritumoral counts of podoplanin-positive lymphatic vessel (Supplementary Fig. 3C and D). Remodeling of the microvasculature was identified by a-SMA labeling of pericytes. All MTCs contained numerous intra- and peritumoral microvessels that were a-SMA positive. Mean a-SMA-positive vessel counts in zone $T$ were significantly higher than those observed in zone $\mathrm{T} / \mathrm{N}$, and the difference was even more significant relative to findings in zone $\mathrm{N}$ (Fig. 5A). Mean intratumoral a-SMA-positive vessel counts in RETmut tumors were significantly higher than those of the RETwt subgroup (Fig. 5B). Again, this was mainly the result of differences between the RETmut and RASmut subgroups (Supplementary Fig. 4). Figure 6 shows an example of CD31, podoplanin and a-SMA staining in a RETmut MTC. There were no significant differences between the three MTC genotype groups in terms of a-SMA-positive vascular cell counts in zones $\mathrm{T} / \mathrm{N}$ and $\mathrm{N}$ (Fig. 5B and Supplementary Fig. 4). Considered separately, however, RASmut T/N counts were lower than those for RETwt/RASwt tumors (Supplementary Fig. 4).

\section{Discussion}

A rich vascular supply is essential for tumor growth and metastasis. This need is met in part by the generation of new blood vessels from existing vascular networks (Turner et al. 2003, Roca \& Adams 2007, Sakurai \& Kudo 2011). Angiogenesis is a very complex process mediated by the coordinated activities of stimulatory and inhibitory factors. Published data on the expression of these factors in MTC are scarce, and most regard a limited set of proangiogenic proteins (generally, VEGF, PDGFRb and VEGFR1, 2 and 3) (Rodriguez-Antona et al. 2013, Mancikova et al. 2014). No attempt has been made to quantitatively assess angiogenesis activation in MTCs as a function of the tumor's mutation profile.

Our findings indicate that the angiogenesis that occurs in MTCs harboring RET mutations may be more intense and complete than that occurring in MTCs with wild-type RET. This conclusion is supported by our analysis of transcript levels in MTCs of genes encoding key components of important pathways involved in the enhancement of tumoral blood supplies. The genes that were upregulated play roles in all the main steps of angiogenesis: I. the induction of endothelial cell proliferation and migration and the enhancement of their survival (VEGF pathway); II. detachment of pericytes and vascular smooth muscle cells from preexisting vessels (angiopoietin 2); III. remodeling of the extracellular matrix (metalloproteases and their tissue inhibitors); IV. Notch pathway activation (ligands, receptors, regulators and effectors), which is indispensable for well-oriented vascular growth characterized by vessels without functional lumens and the absence of excessive sprouting; and finally, V. the maturation and stabilization of the new vessels through the recruitment of pericytes (PDGF pathway, NOTCH3 and angiopoietin 1) (Mitchell \& Parangi 2005, Carmeliet et al. 2009, Phng \& Gerhardt 2009, Raza et al. 2010, Sakurai \& Kudo 2011, Garcia \& Kandel 2012, Thomas et al. 2013). Almost all the proangiogenic genes we analyzed displayed significantly higher mRNA levels in the RETmut tumors than in those with wild-type RET, and the involvement of RET activation in this upregulation was confirmed by the results observed when the gene was silenced with siRNA in MTC cell lines. In vitro experiments showed that in MTC cells, oncogenic RET mutations can activate VEGFRs and PDGFRs, but they probably do not activate Notch signaling, as demonstrated by the stable expression levels after RET knockdown of all the Notch

Published by Bioscientifica Ltd 
pathway effector genes tested (except HES5 in MZ-CRC1 cells). Interestingly, however, RET activation induced the expression of NOTCH ligands, DLL4 and JAG1, raising the possibility that MTC cells themselves might contribute to angiogenesis by activating Notch signaling in nearby endothelial cells.

IHC revealed that all the MTCs were characterized by significantly higher expression of all the proangiogenic proteins considered and increased MVDs in the tumor itself, as compared with corresponding peritumoral and normal thyroid tissues. No genotype-related differences were detected in the intratumoral expression levels of VEGFR2, VEGFR3 or PDGFRa. In contrast, the expression of VEGFR1, PDGFB and NOTCH3 was significantly upregulated in the RETmut cancers (vs those in the RETwt group), confirming the picture that emerged from our transcriptional analysis. (Again, these differences were especially marked when RETmut MTCs were compared with the subset of RETwt tumors that harbored mutations involving RAS). PDGFB and NOTCH3 are mainly involved in the recruitment of pericytes, and their overexpression in RETmut MTCs is in line with our IHC data on the expression of a-SMA, a common pericyte marker (Stapor et al. 2014). Although MVDs in RETmut and RETwt tumors were similar, those in the former group contained higher numbers of intratumoral microvessels that were covered by pericytes. The essential roles played by pericytes in vessel sprouting and the maturation and stabilization of new vessels are well established (Baluk et al. 2003, Bergers \& Song 2005, Ribatti et al. 2011, Ribeiro \& Okamoto 2015). Collectively, therefore, our findings suggest that RETmut MTCs may be characterized by more intense activation of angiogenesis and that this oncogene exerts particularly powerful effects on the remodeling of the tumoral microvasculature, which may be associated with variations in its function (Eberhard et al. 2000, Morikawa et al. 2002, Baluk et al. 2003, Azzi et al. 2013). The differences in angiogenesis state observed between RETmut and RASmut tumors are difficult to explain since RAS itself is activated by RET. It is important to consider, however, that RET can also be activted by pathways other than the MAPK and PI3K cascades, as recently reported by Bagheri-Yarmand and coworkers (Bagheri-Yarmand et al. 2015).

These findings have potentially important implications in this era of personalized medicine, in which the genetic signatures of tumors are used to inform treatment choices (Longo 2012). Our data suggest that the RET mutation status of an MTC could conceivably influence the tumor's response to antiangiogenic drugs, such as TKIs.
If, as we hypothesize, activation of angiogenesis in RETmutated tumors is particularly intense and complete, it is reasonable to speculate that these tumors might also be particularly responsive to TKI-mediated repression of this process, as compared with RET wild-type MTCs, including those with RAS mutations. Only one study has compared in vivo TKI efficacies in patients with RETmut and RASmut tumors. The drug in this case, cabozantinib, produced similar response rates in both groups of tumors, although progression-free survival was appreciably (but not significantly) longer in the RETmut group (60 weeks vs 47 weeks in the RASmut group) (Sherman et al. 2013). It is important to note, however, that the response to cabozantinib might also be influenced by the presence of pericytes, which are not targeted by this drug and appear to be more abundant on the blood vessels of RETmut tumors. The assumption that ablation of these cells renders tumor vessels more accessible to anti-VEGF drugs and consequently more sensitive to their effects (Ribatti et al. 2011) is supported by data generated in several experimental models, which show that combined antiPDGF/anti-VEGF therapy produces greater tumor vessel regression than anti-VEGF therapy alone (Bergers et al. 2003, Erber et al. 2003, Bergers \& Song 2005, Raza et al. 2010). If this assumption is correct, one might reasonably expect this combination therapy to produce particularly obvious benefits in RETmut MTCs than in those without these mutations. Indirect support for this hypothesis has emerged from a recent study on metastatic clear-cell renal cell carcinomas, which showed that the subset of tumors whose vasculatures displayed high pericyte coverage tumors responded better than tumors without this feature to treatment with sunitinib, a multikinase inhibitor with strong effects on both PDGFRb and VEGFRs (Kim et al. 2014). Additional preclinical evidence is needed, however, before this hypothesis can be adequately evaluated.

\section{Supplementary data}

This is linked to the online version of the paper at http://dx.doi.org/10.1530/ ERC-16-0132.

\section{Declaration of interest}

The authors declare that there is no conflict of interest that could be perceived as prejudicing the impartiality of the research reported.

\section{Funding}

The study was supported by the Umberto Di Mario Foundation and the Banca d'Italia, and in part by the grant RF-2011-02350857 from the Italian Ministry of Health (to G T). Amélie Boichard was a recipient of a grant

Published by Bioscientifica Ltd 
from the Association pour la Recherche sur le Cancer (ARC). Valeria Pecce and Francesca Rosignolo contributed to this paper as candidates in the PhD program of Biotechnologies and Clinical Medicine of the Sapienza University of Rome.

\section{Author contribution statement}

A Verrienti, G Tallini and Chiara Colato contributed equally to this work.

\section{Acknowledgments}

Writing support was provided by Marian Everett Kent, BSN (European Medical Writers Association).

\section{References}

Azzi S, Hebda JK \& Gavard J 2013 Vascular permeability and drug delivery in cancers. Frontiers in Oncology 3 211-211. (doi:10.3389/ fonc.2013.00211)

Bagheri-Yarmand R, Sinha KM, Gururaj AE, Ahmed Z, Rizvi YQ Huang SC, Ladbury JE, Bogler O, Williams MD, Cote GJ, et al. 2015 A novel dual kinase function of the RET proto-oncogene negatively regulates activating transcription factor 4-mediated apoptosis. Journal of Biological Chemistry 290 11749-11761. (doi:10.1074/ jbc.M114.619833)

Baluk P, Morikawa S, Haskell A, Mancuso M \& McDonald DM 2003 Abnormalities of basement membrane on blood vessels and endothelial sprouts in tumors. American Journal of Pathology 163 1801-1815. (doi:10.1016/S0002-9440(10)63540-7)

Bergers G \& Song S 2005 The role of pericytes in blood-vessel formation and maintenance 1. Neuro-Oncology 7 452-464. (doi:10.1215/ S1152851705000232)

Bergers G, Song S, Meyer-morse N, Bergsland E \& Hanahan D 2003 Benefits of targeting both pericytes and endothelial cells in the tumor vasculature with kinase inhibitors. Journal of Clinical Investigation 111 1287-1295. (doi:10.1172/JCI200317929)

Boichard A, Croux L, Ghuzlan AA, Broutin S, Dupuy C, Leboulleux S, Schlumberger M, Bidart JM \& Lacroix L 2015 Somatic RAS mutations occur in a large proportion of sporadic RET -negative medullary thyroid carcinomas. Journal of Clinical Endocrinology and Metabolism 97 2031-2035. (doi:10.1210/jc.2012-2092)

Carmeliet P, Smet FD, Loges S \& Mazzone M 2009 Branching morphogenesis and antiangiogenesis candidates: tip cells lead the way. Nature Reviews. Clinical Oncology 6 315-326. (doi:10.1038/ nrclinonc.2009.64)

Castellone MD, Verrienti A, Magendra Rao D, Sponziello M, Fabbro D, Muthu M, Durante C, Maranghi M, Damante G, Pizzolitto S, et al. 2010 A novel de novo germ-line V292M mutation in the extracellular region of RET in a patient with phaeochromocytoma and medullary thyroid carcinoma: functional characterization. Clinical Endocrinology 73 529-534. (doi:10.1111/j.1365-2265.2009.03757.x)

Chau NG \& Haddad RI 2013 Vandetanib for the treatment of medullary thyroid cancer. Clinical Cancer Research 19 524-530. (doi:10.1158/1078-0432.CCR-12-2353)

Ciampi R, Mian C, Fugazzola L, Cosci B, Romei C, Barollo S, Cirello V, Bottici V, Marconcini G, Rosa PM, et al. 2013 Evidence of a low prevalence of RAS mutations in a large medullary thyroid cancer series. Thyroid 23 1-4. (doi:10.1089/thy.2013.2301.ob)

de Groot JWBD, Menacho IP, Schepers H, Osinga J, Eggen BJL \& Hofstra RMW 2006 Cellular effects of imatinib on medullary thyroid cancer cells harboring multiple endocrine neoplasia Type 2A and 2B associated RET mutations. Surgery 139 806-814. (doi:10.1016/ j.surg.2005.10.019)
Deshpande H, Roman S, Thumar J \& Sosa JA 2011 Vandetanib (ZD6474) in the treatment of medullary thyroid cancer. Clinical Medicine Insights Oncology 5 213-221. (doi:10.4137/cmo.s6197)

Durante C \& Filetti S 2011 Management of papillary thyroid cancer patients in absence of postoperative radioiodine remnant ablation: tailoring follow-up by neck sonography. Journal of Clinical Endocrinology and Metabolism 96 3059-3061. (doi:10.1210/jc.2011-1379)

Eberhard A, Kahlert S, Goede V, Hemmerlein B, Plate KH \& Augustin HG 2000 Heterogeneity of angiogenesis and blood vessel maturation in human tumors: implications for antiangiogenic tumor therapies. Cancer Research 60 1388-1393.

Erber R, Thurnher A, Katsen AD, Groth G, Kerger H, Hammes H-P, Menger MD, Ullrich A \& Vajkoczy P 2003 Combined inhibition of VEGF- and PDGF-signaling en- forces tumor vessel regression by interfering with pericyte- mediated endothelial cell survival mechanisms. FASEB Journal 18 338-340. (doi:10.1096/fj.03-0271fje)

Ferreira CV \& Maia AL 2013 Advanced medullary thyroid cancer: pathophysiology and management. Cancer Management and Research 5 57-66. (doi:10.2147/cmar.s33105)

Garcia A \& Kandel JJ 2012 Notch: a key regulator of tumor angiogenesis and metastasis. Histology and Histopathology 27 151-156. (doi:10.14670/HH-27.151)

Kim BH, Sohn JC, Ha JY, Park CH, Choe MS, Jung HR \& Kim CI 2014 Relationships between the effect of sunitinib and immature blood vessels in metastatic renal cell cancer. Urologia Internationalis 94 137-143. (doi:10.1159/000363773)

Longo DL 2012 Tumor heterogeneity and personalized medicine. New England Journal of Medicine 366 883-892. (doi:10.1056/ NEJMe1200656)

Mancikova V, Kersten I, Leandro-garcı LJ, Sa L, Sastre-marcos J, Garcı JF \& Rodrı C 2014 Expression is influenced by RAS mutations in medullary thyroid carcinoma. Thyroid 24 1251-1255. (doi:10.1089/ thy.2013.0579)

Mitchell JC \& Parangi S 2005 Angiogenesis in benign and malignant thyroid disease. Thyroid 15 494-510. (doi:10.1089/thy.2005.15.494)

Morikawa S, Baluk P, Kaidoh T, Haskell A, Jain RK \& McDonald DM 2002 Abnormalities in pericytes on blood vessels and endothelial sprouts in tumors. American Journal of Pathology 160 985-1000. (doi:10.1016/S0002-9440(10)64920-6)

Phng L \& Gerhardt H 2009 Review angiogenesis: a team effort coordinated by notch. Developmental Cell 16 196-208. (doi:10.1016/ j.devcel.2009.01.015)

Puppin C, Durante C, Sponziello M, Verrienti A, Pecce V, Lavarone E, Baldan F, Campese AF, Boichard A, Lacroix L, et al. 2014 Overexpression of genes involved in miRNA biogenesis in medullary thyroid carcinomas with RET mutation. Endocrine 47 528-536. (doi:10.1007/s12020-014-0204-3)

Pusztaszeri MP, Bongiovanni M \& Faquin WC 2014 Update on the cytologic and molecular features of medullary thyroid carcinoma. Advances in Anatomic Pathology 21 26-35. (doi:10.1097/ PAP.0000000000000004)

Raza A, Franklin MJ \& Dudek AZ 2010 Critical review pericytes and vessel maturation during tumor angiogenesis and metastasis. American Journal of Hematology 85 593-598. (doi:10.1002/ajh.21745)

Ribatti D, Nico B \& Crivellato E 2011 The role of pericytes in angiogenesis. International Journal of Developmental Biology $\mathbf{5 5}$ 261-268. (doi:10.1387/ijdb.103167dr)

Ribeiro AL \& Okamoto OK 2015 Combined effects of pericytes in the tumor microenvironment. Stem Cells International 2015 1-8. (doi:10.1155/2015/868475)

Roca C \& Adams RH 2007 Regulation of vascular morphogenesis by Notch signaling. Genes and Development 21 2511-2524. (doi:10.1101/ gad.1589207)

Rodriguez-Antona C, Munoz-Repeto I, Inglada-Perez L, de Cubas AA, Mancikova V, Canamero M, Maliszewska A, Gomez A, Leton R, Leandro-Garcia LJ, et al. 2013 Influence of RET mutations on the 
expression of tyrosine kinases in medullary thyroid carcinoma. Endocrine-Related Cancer 20 611-619. (doi:10.1530/ERC-12-0316)

Sakurai T \& Kudo M 2011 Signaling pathways governing tumor angiogenesis. Oncology 81 24-29. (doi:10.1159/000333256)

Sherman SI, Cohen EEW, Schoffski P, Elisei R, Schlumberger M, Wirth LJ, Mangeshkar M, Aftab DT, Clary DO \& Brose MS 2013 Efficacy of cabozantinib (Cabo) in medullary thyroid cancer (MTC) patients with RAS or RET mutations: results from a phase III study. Journal of Clinical Oncology 31 (May 20 Supplement) abstract 6000. (available at: http://meeting.ascopubs.org/cgi/ content/abstract/31/15_suppl/6000)

Sponziello M, Durante C, Boichard A, Dima M, Puppin C, Verrienti A, Tamburrano G, Di Rocco G, Redler A, Lacroix L, et al. 2014 Epigenetic-related gene expression profile in medullary thyroid cancer revealed the overexpression of the histone methyltransferases EZH2 and SMYD3 in aggressive tumours. Molecular and Cellular Endocrinology 392 8-13. (doi:10.1016/j.mce.2014.04.016)

Stapor PC, Sweat RS, Dashti DC, Betancourt AM \& Murfee WL 2014 Pericyte dynamics during angiogenesis: new insights from new identities. Journal of Vascular Research 51 163-174. (doi:10.1159/000362276)
Tallini G, Ghossein RA, Emanuel J, Gill J, Kinder B, Dimich AB, Costa J, Robbins R, Burrow GN \& Rosai J 1998 Detection of thyroglobulin, thyroid peroxidase, and RET/PTC1 mRNA transcripts in the peripheral blood of patients with thyroid disease. Journal of Clinical Oncology 16 1158-66.

Thomas J-L, Aphp F, Pitié-salpètrière GH, Baker K, Pierre U, Paris MC, Nurmi H \& France CD 2013 Interactions between VEGFR and Notch signaling pathways in endothelial and neural cells. Cellular and Molecular Life Sciences 70 1779-1792. (doi:10.1007/ s00018-013-1312-6)

Turner HE, Harris AL, Melmed S \& Wass JAH 2003 Angiogenesis in endocrine tumors. Endocrine Reviews 24 600-632. (doi:10.1210/ er.2002-0008)

Wells SA, Asa SL, Dralle H, Elisei R, Evans DB, Gagel RF, Lee N, Machens A, Moley JF, Pacini F, et al. 2015 Revised American Thyroid Association guidelines for the management of medullary thyroid carcinoma. Thyroid 25 567-610. (doi:10.1089/ thy.2014.0335)

Wu LS, Roman SA \& Sosa JA 2011 Medullary thyroid cancer: an update of new guidelines and recent developments. Current Opinion in Oncology 23 22-27. (doi:10.1097/CCO.0b013e328340b527)

Received in final form 23 June 2016

Accepted 7 July 2016

Accepted Preprint published online 7 July 2016 